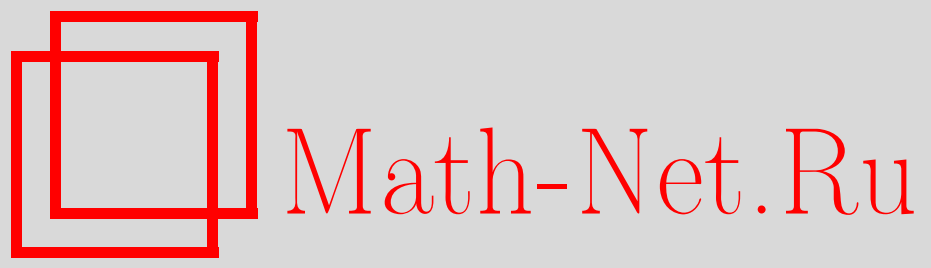

Обцероссийский математический портал

И. В. Соболев, Действие циклических групп на трехмерных многообразиях Фано, Матем. заметки, 2000, том 68, выпуск 5, 793-795

DOI: https://doi.org/10.4213/mzm1001

Использование Общероссийского математического портала Math-Net.Ru подразумевает, что вы прочитали и согласны с пользовательским соглашением http://www.mathnet.ru/rus/agreement

Параметры загрузки:

IP : 34.229 .108 .108

26 апреля 2023 г., 16:51:37 


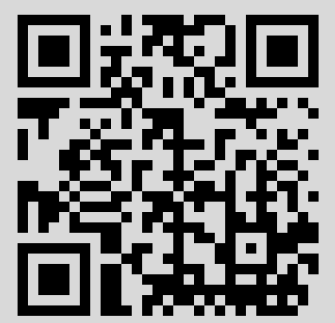




\section{ДЕЙСТВИЕ ЦИКЛИЧЕСКИХ ГРУПП НА ТРЕХМЕРНЫХ МНОГООБРАЗИЯХ ФАНО}

\section{И.В. Соболев}

Из программы минимальных моделей следует, что классификация многообразий Фано с терминальными особенностями важна для описания всех алгебраических многообразий, однако на современном этапе развития алгебраической геометрии об особых многообразиях Фано известно довольно мало.

Кавамата доказал, что существует лиш конечное (с точностью до деформаций) число типов трехмерных терминальных многообразий Фано с числом Пикара 1 (см. [1]). Намикава в [2] установил, что любое трехмерное многообразие Фано с терминальными горенштейновыми особенностями является деформацией гладкого.

Этими двумя результатами сведения об общих трехмерных терминальных многообразиях Фано фактически исчерпьваются.

Существует классификация некоторых частных типов многообразий рассматриваемого класса. Например, Сано в работе [3] перечис лил все трехмерные многообразия Фано индекса 1 с терминальньми циклическими факторособенностями. Аналогичная техника (изучение инволюций на гладких многообразиях Фано) была использована Бейлем для классификации трехмерных многообразий с гиперплоскими сечениями - поверхностями Энриквеса (см. [4]).

Настоящая работа является попыткой перенести результаты [3] и [4] на действие циклических групп любого порядка. Мы получаем следующую теорему.

Пусть $X$ - гладкое трехмерное многообразие $с h^{i}\left(X, O_{X}\right)=0$ для всех $i>0$, причем на $X$ действует группа $\mathbb{Z}_{p}(p$ - простое $)$ так, что фактормногообразие $X / \mathbb{Z}_{p}$ имеет терминальные особенности. Тогда $p+1$ делит 24, причем $е(X) \equiv 24 /(p+1)(\bmod p)$ $(e(x)$ - эйлерова характеристика $X)$.

Для доказательства нам понадобятся три факта.

1. Голоморфная формула Лефшеца. Пусть автоморфизм $\sigma$ действует на компактном комплексном многообразии $X$, имея лишь изолированные неподвижные точки. Тогда

$$
\sum_{\sigma(x)=x}\left[\operatorname{det}\left(\operatorname{Id}-(d \sigma)_{x}\right)\right]^{-1}=\sum_{q}(-1) \operatorname{Tr}\left(\left.\sigma^{*}\right|_{H^{q}\left(X, O_{X}\right)} \quad\right. \text { (см. [5]). }
$$

2. Классификация трехмерных терминальных факторособенностей. Если $(X, P)$ - такая осо-

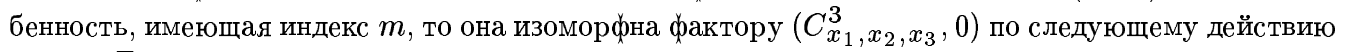
группы $\mathbb{Z}_{m}$ :

$$
\left(x_{1}, x_{2}, x_{3}\right) \rightarrow\left(\varepsilon^{a} x_{1}, \varepsilon^{-a} x_{2}, \varepsilon^{b} x_{3}\right),
$$

где $\varepsilon$ - примитивный корень из 1 степени $m, a$ и $b$ взаимно просты с $m$ (cм. [6]).

3. В алгебраической топологии известно много результатов, относящихся к действию циклических групп на многообразиях. Из них мы исползуем следующий (см. [7]): пусть на компактном

(C) И.В. Соболев 2000 
комплексном многообразии $X$ действует автоморфизм порядка $p$ ( $p$ - простое), имеющий $q$ неподвижных точек. Тогда $e(X) \equiv q(\bmod p)$.

Перейдем теперь к доказательству теоремы.

Лемма 1. Пусть $\varepsilon^{p}=1, \varepsilon \neq 1, p-$ nростое. Тогда

1) $(1-\varepsilon)^{-1}=-\frac{1}{p} \sum_{k=1}^{p-1} k \varepsilon^{k}$

2) $е с л u \sum_{k=0}^{p-1} C_{k} \varepsilon^{k}=0$, əде $C_{k} \in \mathbb{Z}$, mo $C_{0}=\cdots=C_{p-1}$.

ДокАЗАТЕЛЬСтво. 1) Проверяется прямым вычислением.

2) Так как многочлен $1+x+\cdots+x^{p-1}$ неприводим над $\mathbb{Q}$, то $\varepsilon, \varepsilon^{2}, \ldots, \varepsilon^{p-1}$ линейно независимы над $\mathbb{Q}$, значит, 1 однозначно представляется в виде линейной комбинации $\varepsilon, \varepsilon^{2}, \ldots, \varepsilon^{p-1} \mathrm{c}$ рациональными коэффициентами.

ЛЕмма 2. Для любого простого $p$ и $a, b$, не делящихся на $p$,

$$
S(a, b, p)=\sum_{\substack{a(k-l)+b m \equiv 0(\bmod p) \\ 0 \leqslant k, l, m \leqslant p-1}} k l m=\frac{p}{2}\left[\frac{p^{2}(p-1)^{2}}{4}-\frac{p(p-1)(2 p-1)}{6}\right] .
$$

ДоКАЗАТЕЛЬСтво. Если тройка $(k, l, m)$ входит в сумму $S(a, b, p)$, то в нее входит и тройка $(l, k, p-m)$. Ясно также, что для любых $k, l$ существует единственное $m$ такое, что $0 \leqslant m \leqslant p-1$ и $a(k-l)+b m \equiv 0(\bmod p)$. Поэтому

$$
\begin{aligned}
S(a, b, p) & =\sum_{1 \leqslant k<l \leqslant p-1} p k l=\frac{p}{2}\left[\sum_{1 \leqslant k, l \leqslant p-1} k l-\sum_{k=1}^{p-1} k^{2}\right] \\
& =\frac{p}{2}\left[\frac{p^{2}(p-1)^{2}}{4}-\frac{p(p-1)(2 p-1)}{6}\right] .
\end{aligned}
$$

Пусть теперь $X$ - многообразие, удовлетворяющее условиям теоремы. Пусть $\sigma$ - автоморфизм $X$, соответствующий образующей группы $\mathbb{Z}_{p}$. Тогда в касательном пространстве к неподвижной точке $x(d \sigma)_{x}$ приводится к виду

$$
\left(\begin{array}{lll}
\varepsilon^{a} & & \\
& \varepsilon^{-a} & \\
& & \varepsilon^{b}
\end{array}\right)
$$

где $\varepsilon^{p}=1, \varepsilon \neq 1, a, b$ не делятся на $p$. В этом случае будем говорить, что точка $x$ имеет тип $(a, b)$.

Обозначим через $N_{a, b}$ число точек типа $(a, b)$. Так как $h^{i}\left(X, O_{X}\right)=0$ при $i>0$, то из голоморфной формулы Лефшеца следует, что

$$
\sum_{a, b} N_{a, b}\left[\left(1-\varepsilon^{a}\right)\left(1-\varepsilon^{-a}\right)\left(1-\varepsilon^{b}\right)\right]^{-1}=1 .
$$

По лемме 1.1) это равенство можно переписать так:

$$
-\frac{1}{p^{3}} \sum_{n=0}^{p-1}\left(\sum_{a, b} N_{a, b} \sum_{a(k-l)+b m \equiv n(\bmod p)} k l m\right) \varepsilon^{n}=1 .
$$

В силу леммы 1.2) это значит, что

$$
\sum_{a, b} N_{a, b} \sum_{a(k-l)+b m \equiv n(\bmod p)} k l m=\sum_{a, b} N_{a, b} \sum_{a(k-l)+b m \equiv 0(\bmod p)} k l m+p^{3}
$$


для всех $n \neq 0$ или

$$
\sum_{a, b} N_{a, b}\left(\sum_{a(k-l)+b m \equiv n(\bmod p)} k l m-\sum_{a(k-l)+b m \equiv 0(\bmod p)} k l m\right)=p^{3} .
$$

Складывая эти $p-1$ уравнений, получаем

$\sum_{n=1}^{p-1} \sum_{a, b} N_{a, b} \sum_{a(k-l)+b m \equiv n(\bmod p)} k l m-(p-1) \sum_{a, b} N_{a, b} \sum_{a(k-l)+b m \equiv 0(\bmod p)} k l m=p^{3}(p-1)$.

Переставляя знаки суммирования, будем иметь

$$
\sum_{a, b} N_{a, b} \sum_{n=0}^{p-1} \sum_{a(k-l)+b m \equiv n(\bmod p)} k l m-p \sum_{a, b} N_{a, b} \sum_{a(k-l)+b m \equiv 0(\bmod p)} k l m=p^{3}(p-1) .
$$

Очевидно, что

$$
\sum_{n=0}^{p-1} \sum_{a(k-l)+b m \equiv n(\bmod p)} k l m=\sum_{1 \leqslant k, l, m \leqslant p-1} k l m=\frac{p^{3}(p-1)^{3}}{8} .
$$

По лемме 2

$$
\sum_{a(k-l)+b m \equiv 0(\bmod p)} k l m=\frac{p}{2}\left[\frac{p^{2}(p-1)^{2}}{4}-\frac{p(p-1)(2 p-1)}{6}\right] .
$$

Окончательно имеем

$$
\sum_{a, b} N_{a, b}=\frac{24}{p+1}
$$

Отсюда следует утверждение теоремы.

Наш результат позволяет классифицировать терминальные многообразия Фано, получающиеся факторизацией гладких по циклической группе. Нужно перебрать все гладкие многообразия Фано, которые принадлежат известному конечному списку, и исследовать автоморфизмы порядка $p$, имеющие ровно $24 /(p+1)$ неподвижных точек. Условие на эйлерову характеристику существенно сокращает перебор при $p \neq 2$.

\section{СПИСОК ЦИТИРОВАННОЙ ЛИТЕРАТУРЫ}

1. Kawamata Y. // Contemp. Math. 1992. V. 131. P. 439-445. 2. Namikawa Y. // J. Alg. Geom. 1997. V. 6. № 2. P. 307-324. 3. Sano T. // J. Math. Soc. Japan. 1995. V. 47. № 2. P. 369-380. 4. Bayle L. // J. Reine Angew. Math. 1994. V. 449. P. 9-63. 5. Гриффитс Ф., Харрис Дж. Принципы алгебраической геометрии. Т. 1. М.: Мир, 1982. 6. Данилов В. И. // Изв. АН СССР. Сер. Матем. 1982. Т. 46. С. 971-982. 7. Панов Т. Е. // Изв. РАН. Сер. Матем. 1998. Т. 62. № 3. C. $87-120$. 